Check for updates

Cite this: RSC Adv., 2018, 8, 13813

\title{
POSS-enhanced thermosensitive hybrid hydrogels for cell adhesion and detachment $\uparrow$
}

\author{
Yudong Tong, ${ }^{a}$ Yuanhao Zhang, ${ }^{\mathrm{b}}$ Yangyang Liu, ${ }^{\mathrm{a}}$ Haibo Cai, ${ }^{\text {a }}$ Weian Zhang (D) *b \\ and Wen-Song Tan ${ }^{a}$
}

Thermosensitive poly( $N$-isopropylacrylamide) (PNIPAM)-based substrates have presented great promise in cell sheet engineering. However, non-functionalized PNIPAM cannot be well applied for cell cultivation, due to the low cell adhesion. Herein, to enhance PNIPAM-based substrates and to promote cell proliferation and detachment, a polyhedral oligomeric silsesquioxane (POSS) nanoscale inorganic enhanced agent has been introduced into PNIPAM matrices to construct POSS-containing hybrid hydrogels. The hydrogels were facilely prepared using POSS as a cross-linker via one-pot crosslinking reaction under UV irradiation. The swelling behavior, thermal stability and the mechanical properties of POSS-PNIPAM hybrid hydrogels have been evaluated and they are all dependent on the content of POSS. The in vitro experiment confirms that human amniotic mesenchymal stem cells (hAMSCs) exhibit clearly enhanced adhesion and proliferation on the substrates of POSS-PNIPAM hybrid hydrogels in comparison to the pure PNIPAM hydrogel without POSS. Based on the thermal-responsiveness of PNIPAM, the proliferated cells are easily released without damage from the surface of hybrid hydrogels. Therefore, POSS-enhanced PNIPAM hybrid hydrogels provide a unique approach for harvesting anchorage dependent stem cells.

Received 22nd February 2018

Accepted 5th April 2018

DOI: $10.1039 / \mathrm{c} 8 \mathrm{ra0} 01584 \mathrm{~h}$

rsc.li/rsc-advances responsive property could be tuned in specific range by varying the composition and structure of the smart materials. Among those, thermosensitive materials are the most widely used for harvesting cells, since their thermo-sensitivity could be easily and precisely accomplished both in vitro and in vivo. ${ }^{15-17}$ As a typical thermo-responsive material, $\operatorname{poly}(\mathrm{N}$-isopropylacrylamide) (PNIPAM) has been well studied since it has a reversible phase transition at the lower critical solution temperature (LCST) in aqueous solution. ${ }^{18,19}$ When the temperature below the LCST, PNIPAM is hydrated with the chains extended, due to the presence of hydrogen bonding in aqueous solution. While above the LCST, PNIPAM chains shrink and the surface becomes hydrophobic. Based on this specific property of PNIPAM, PNIPAM-based thermosensitive materials have been fabricated for the harvesting stem cells. For example, Okano et al. grafted PNIPAM onto the commercial polystyrene plate surface for cell culture, and then the cultured cells were detached and harvested by decreasing the temperature below LCST of PNIPAM. ${ }^{20} \mathrm{Li}$ et al. constructed a dualresponsive hydrogel based on thermosensitive PNIPAM copolymers with phenylboronic acid group, and further applied for the cell harvest. The cells could be well released at a lower temperature and high sugar concentration. ${ }^{21}$ Additionally, Yu et al. fabricated a nano-patterned PNIPAM surface by using an interferometric lithography (IL) technique, and they found that nano-patterning and thickness of PNIPAM-based substrates were all crucial factors for cell proliferation and harvest. ${ }^{22}$
${ }^{a}$ State Key Laboratory of Bioreactor Engineering, East China University of Science and Technology, 130 Meilong Road, Shanghai 200237, P. R. China. E-mail: caihaibo@ ecust.edu.cn; Fax: +86 21 64253033; Tel: +862164253033

${ }^{b}$ Shanghai Key Laboratory of Functional Materials Chemistry, East China University of Science and Technology, 130 Meilong Road, Shanghai 200237, P. R. China. E-mail: wazhang@ecust.edu.cn

$\uparrow$ Electronic supplementary information (ESI) available: Experimental details and additional data. See DOI: 10.1039/c8ra01584h 
Although PNIPAM with a superior thermo-responsiveness has exhibited a great promising for cell harvest, nonfunctionalized PNIPAM-based surfaces are not suitable for cell attachment, leading to a low efficiency of cell harvest. During the cell culture, the attachment of cells is a complicated process, which is often determined by the properties of substrates including the thickness, ${ }^{22}$ surface charge, ${ }^{23}$ topology, ${ }^{24,25}$ stiffness $^{26,27}$ and biocompatibility. ${ }^{28}$ Recently, organic/inorganic hybrid materials have been constructed for cell culture, and the results showed the introduction of inorganic components such as carbon nanotubes, ${ }^{29}$ gold nanoparticles $^{30}$ and graphene oxide ${ }^{31}$ could efficiently enhance the cell attachment. For example, Chen et al. prepared MWCNTsinterpenetrated PNIPAM hydrogels, and they demonstrated that enhanced mechanical strength and hydrophobicity of hybrid hydrogels could well promote cell attachment. ${ }^{29} \mathrm{HeO}$ et al. developed a novel functional hydrogel with gold nanoparticles (GNPs), the in vitro experiments showed that the reinforced composite hydrogels are suitable for stem cell differentiation control and bone tissue regeneration. ${ }^{30}$ More recently, polyhedral oligomeric silsesquioxane (POSS) has also been used to construct POSS-containing hybrid hydrogels with improved performance for cell culture. ${ }^{32}$ Our previous work showed POSS-based poly(ethylene glycol) (PEG) hybrid hydrogels prepared via azide-alkyne click reaction could effectively enhance the adherence and proliferation of chondrocytes on hybrid hydrogels. ${ }^{33}$ However, to the best of our knowledge, POSS-based thermosensitive PNIPAM hybrid hydrogel has not been developed for cell attachment and detachment.

In this work, we construct a POSS-containing PNIPAM thermo-sensitive hydrogel via photo-crosslinking reaction by using octa-methacrylated POSS as a cross-linker. Here, the introduction of POSS moieties allows the hybrid hydrogel with the following merits: (1) octa-methacrylated POSS with a defined molecular structure as a cross-linker can be well incorporated into PNIPAM matrices to form a homogeneous hydrogel; (2) POSS is one of the smallest inorganic enhanced agents, which can significantly improve the stiffness of substrates; ${ }^{\mathbf{1 , 3 4 , 3 5}}$ (3) POSS has been confirmed to be nontoxic and biocompatible, which could improve the biocompatibility of POSS-PNIPAM hybrid hydrogels. ${ }^{36-38}$ To well reveal above advantages of POSS-PNIPAM hybrid hydrogels, their morphologies and properties have been discussed by varying the POSS content. Human amniotic mesenchymal stem cells (hAMSCs) are cultured on these hybrid hydrogels, and enhanced cell attachment and detachment are further evaluated.

\section{Experimental}

\subsection{Materials}

NIPAM was supplied by Sigma-Aldrich (St. Louis, MO, USA) and further purified by recrystallization from a mixed solution of toluene/n-hexane $(6: 1, \mathrm{v} / \mathrm{v})$. OMAPOSS was purchased from Hybrid Plastics (Hattiesburg, MS, USA) and used as received. 1,4-Dioxane and ethanol were purchased from Titan Scientific (Shanghai, China). Irgacure 2959 was purchased from Wako Pure Chemical Industries (Osaka, Japan). Alpha-minimum essential medium ( $\alpha$-MEM) and phosphate buffer saline (PBS) were purchased from HyClone (South Logan, UT, USA). Fetal bovine serum (FBS), penicillin/streptomycin solution $(100 \times)$ and trypsin was purchased from Gibco (Grand Island, NY, USA). Cell counting kit-8 (CCK-8) was purchased from Dojindo (Kumamoto, Japan). Ethylenediamine tetraacetic acid (EDTA), trypan blue, calcein-AM, propidium iodide (PI), $N, N^{\prime}$-methylenebisacrylamide (MBA), OEGMA $\left(M_{\mathrm{n}}=500 \mathrm{~g} \mathrm{~mol} \mathrm{~m}^{-1}\right)$, rhodamine-phalloidin and 4',6-diamidino-2-phenylindole (DAPI) were purchased from Sigma-Aldrich (St. Louis, MO, USA). Distilled water was prepared using a Milli-Q system (EMD Millipore, Billerica, MA, USA) and used in all experiments.

\subsection{Synthesis of POSS-PNIPAM hybrid hydrogels}

POSS-PNIPAM hydrogels were prepared from a photocrosslinking process using POSS as a cross-linker. Typically, NIPAM (135 mg, $1.19 \mathrm{mmol}$ ), OEGMA (18 mg, $0.036 \mathrm{mmol}$ ), Irgacure 2959 ( $4 \mathrm{mg}, 0.018 \mathrm{mmol}$ ) and OMAPOSS (10 mg, 0.007 mmol) were dissolved in $1 \mathrm{~mL}$ of 1,4-dioxane. The pre-gel solution was injected into a PTFE mould with cylindrical recesses: $15.6 \mathrm{~mm} \times 1.0 \mathrm{~mm}$ (diameter $\times$ thickness), then covered with glass sheets. Subsequently, the mould was placed under a UV lamp $\left(\lambda=365 \mathrm{~nm}, 8 \mathrm{~mW}\right.$ per $\left.\mathrm{cm}^{2}\right)$, the distance from the mould to the light source was about $100 \mathrm{~mm}$. To ensure adequate crosslinking, the exposure time of UV irradiation was $30 \mathrm{~min}$. After the reaction, the polymerized hydrogels were soaked in 1,4-dioxane for 2 days to remove unreacted molecules. Then, the solvent was completely replaced with water by washing with Milli-Q water for 3 days. The obtained PNIPAM based hydrogels were saved in $4{ }^{\circ} \mathrm{C}$ Milli-Q water before further use (Scheme 1). With a similar approach, the other two POSSPNIPAM hydrogels were prepared using the different OMAPOSS contents (20 mg, $0.014 \mathrm{mmol} ; 30 \mathrm{mg}, 0.021 \mathrm{mmol}$ ), so these three POSS-PNIPAM hybrid hydrogels can be defined as P10N, $\mathrm{P} 20 \mathrm{~N}$ and $\mathrm{P} 30 \mathrm{~N}$, respectively. Additionally, the control of pure

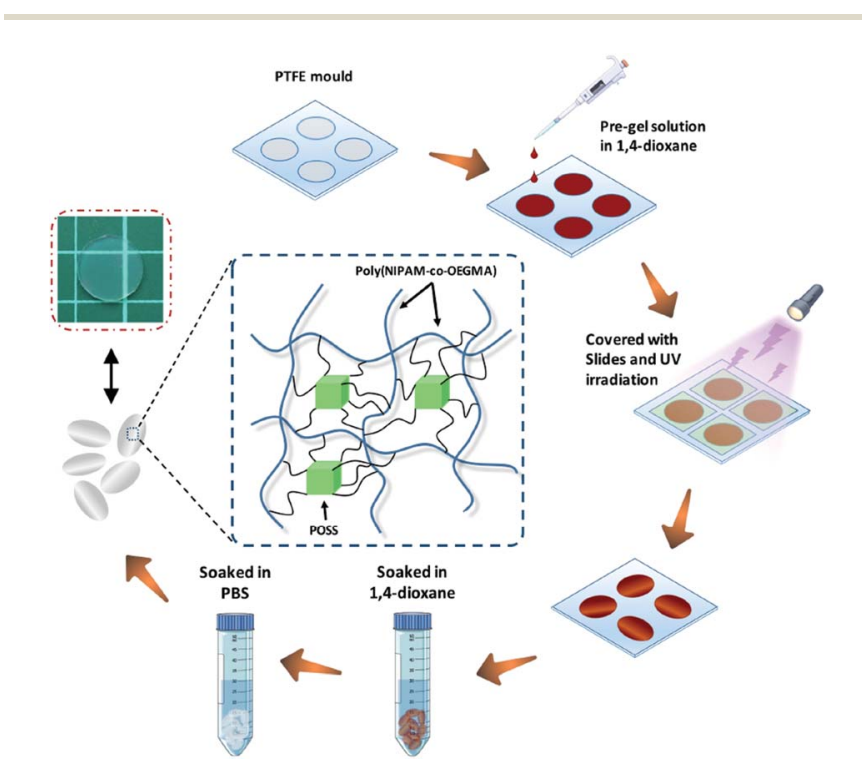

Scheme 1 The fabrication of POSS-PNIPAM hydrogel by UV-initiated crosslinking. 
PNIPAM hydrogel (MN hydrogel) was prepared using $N, N^{\prime}$ methylenebisacrylamide (MBA) as a cross-linker. The synthetic scheme and detail synthetic condition are shown in Fig. S1 and Table S1, $\dagger$ respectively.

\subsection{Characterizations}

Thermogravimetric analysis (TGA). The thermal properties of the PNIPAM based hydrogels were measured by a PerkinElmer Pyris-1 thermogravimetric analyzer in nitrogen atmosphere from $40{ }^{\circ} \mathrm{C}$ to $800{ }^{\circ} \mathrm{C}$ at a heating rate of $10^{\circ} \mathrm{C} \mathrm{min}{ }^{-1}$.

Scanning electron microscopy (SEM). The micro morphology of PNIPAM based hydrogels was characterized by a scanning electron microscope (SEM, JSM-6360LV, Jeol). All the hydrogel samples were dehydrated by freeze-drying for $48 \mathrm{~h}$ after swelling equilibrium in PBS $\left(20^{\circ} \mathrm{C}\right)$. Before observation, the fracture surfaces of the freeze-dried hydrogels were sputter-coated with a thin layer of gold for 2 min and imaged at an accelerating voltage of $10 \mathrm{kV}$.

\subsection{Temperature-dependent equilibrium swelling}

The freeze-dried POSS-PNIPAM hybrid hydrogel samples were immersed in $\mathrm{PBS}$ at $4{ }^{\circ} \mathrm{C}$ as the initial temperature. The temperature was slowly increased to $50{ }^{\circ} \mathrm{C}$ with a heat rate of $0.5{ }^{\circ} \mathrm{C} \mathrm{min}{ }^{-1}$ at intervals of about $4{ }^{\circ} \mathrm{C}$. At each predetermined temperature interval, the hydrogel samples were equilibrated for $15 \mathrm{~min}$ to reach swelling equilibrium, and then the weight was measured after removing excess surface deionized water. The swelling ratio (SR) was calculated by the following formula:

$$
\mathrm{SR}=\left(W_{\text {swell }}-W_{\text {dry }}\right) / W_{\text {dry }} \times 100 \%
$$

where $W_{\text {swell }}$ and $W_{\text {dry }}$ are the weight of the swollen and freezedried hydrogels, respectively.

\subsection{Mechanical characterization of POSS-PNIPAM hybrid hydrogels}

The unconfined compression testing was performed by using a mechanical testing system (GT-TCS2000, GOTECH, China). The hydrogel films were prepared with dimension of $10 \mathrm{~mm} \times$ $5.0 \mathrm{~mm}$ (diameter $\times$ thickness). All the hydrogel films were compressed with a crosshead at a speed of $1 \mathrm{~mm} \mathrm{~min}^{-1}$ at $20^{\circ} \mathrm{C}$ until fractured. The elastic modulus was calculated according to the initial $10 \%$ portion of the stress-strain curves that was nearly linear. Rheological measurement was performed by using a rotational rheometer (RS600, Thermo Hakke, USA). A special mold was used to fabricate hydrogel disks with dimension of $20 \mathrm{~mm} \times 1.0 \mathrm{~mm}$ (diameter $\times$ thickness). Storage and loss modulus were measured by using a frequency sweep mode with the range from 0.1 to $10 \mathrm{~Hz}\left(\mathrm{rad} \mathrm{s}^{-1}\right)$ at a proper strain (1\%) at $20^{\circ} \mathrm{C}$.

\subsection{Cell culture}

Human amniotic mesenchymal stromal cells (hAMSCs) were accepted as a gift from Professor Y. Zhou (East China University of Science and Technology, Shanghai, China), and were prepared according to procedures described elsewhere. ${ }^{39}$
hAMSCs were cultured in alpha-minimum essential medium ( $\alpha$ MEM) supplemented with $10 \%(\mathrm{v} / \mathrm{v})$ fetal bovine serum (FBS) and $1 \%$ penicillin/streptomycin under a humidified atmosphere with $5 \% \mathrm{CO}_{2}$ at $37{ }^{\circ} \mathrm{C}$. The medium was changed every two days, and the cells were harvested by using $0.25 \%$ trypsin/ EDTA in PBS after reaching sub-confluency.

Before cell cultivation, the POSS-PNIPAM hydrogels were sterilized with alcohol (75\%) for $6 \mathrm{~h}$, and followed by UV irradiation for $2 \mathrm{~h}$. The sterilized gels were rinsed with PBS for $2 \mathrm{~h}$ and then immersed in cell culture medium for $12 \mathrm{~h}$ at $37{ }^{\circ} \mathrm{C}$ before cell seeding. hAMSCs (Passage 6) were seeded on the surface of POSS-PNIPAM hydrogels at a cell density of $2.5 \times 10^{4}$ cells per $\mathrm{cm}^{2}$, and incubated under a humidified atmosphere with $5 \% \mathrm{CO}_{2}$ at $37^{\circ} \mathrm{C}$, and the culture medium was changed every two days. Cell counting kit-8 (CCK-8) assay was performed to evaluate the cell attachment and proliferation on hydrogel surfaces. At each predetermined time interval, the hydrogel films were incubated with $\alpha$-MEM supplemented $10 \%$ (v/v) CCK8 in cell incubator at $37^{\circ} \mathrm{C}$ for $2 \mathrm{~h}$. The optical density values (O.D.) of each supernatant at $450 \mathrm{~nm}$ were measured by a microplate reader (PowerWave XS2, BioTek, USA). Cytoskeleton staining (F-actin \& nuclei) was performed to visualize the morphology of hAMSCs on POSS-PNIPAM hydrogels. After culturing for 4 days, the hydrogel samples were removed from the cell culture plates and the cells were fixed in $4 \%$ paraformaldehyde for $20 \mathrm{~min}$. After that, the samples were soaked in $0.3 \%$ Triton X-100 for $5 \mathrm{~min}$. Then the F-actin and nuclei of cells were stained with rhodamine-phalloidin and DAPI, respectively. Immunofluorescence staining images for F-actin and nuclei of the hAMSCs were obtained by a confocal laser scanning microscope (A1R, Nikon, Japan). Cell harvest was performed at day 4, where the hydrogel films were immersed in $4{ }^{\circ} \mathrm{C}$ fresh medium to be fully swollen. After $40 \mathrm{~min}$, the cells on hydrogels were almost detached, and the process of cell detachment from the POSS-PNIPAM hydrogel by temperature stimulation was illustrated in Scheme 2. Then the supernatant was collected with a centrifuge at $1500 \mathrm{rpm}$ for $5 \mathrm{~min}$, and the trypsin was added to count the cell number. The viability of the harvested cells was assessed by live/dead assay: the harvested cells were reseeded on tissue culture polystyrene (TCPS) or hydrogels at a density of $2.5 \times 10^{4}$ cells per $\mathrm{cm}^{2}$. After culturing for $24 \mathrm{~h}$, the hydrogel films were directly rinsed with the PBS containing $2 \mathrm{mM}$ calcein-AM (live, green) and $2 \mathrm{mM}$ PI (dead, red) in an incubator at $37{ }^{\circ} \mathrm{C}$ for $30 \mathrm{~min}$. The images were obtained by using an inverted fluorescence microscope (Ti-s, Nikon, Japan).

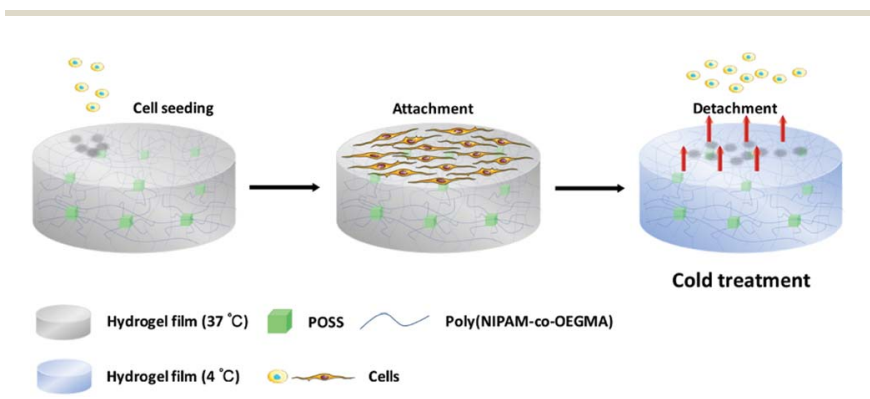

Scheme 2 Schematic illustration of the process for temperatureinduced cell detachment. 


\subsection{Statistical analysis}

Each experiment was performed at least in triplicate $(n=3)$ and the values were denoted as means \pm SD. Statistical significance was evaluated by one-way ANOVA using SPSS Statistics with a level of significance $p<0.05$.

\section{Results and discussion}

\subsection{Synthesis and characterization of POSS-PNIPAM hybrid hydrogels}

POSS-PNIPAM hybrid hydrogels have been reported by several groups. ${ }^{40-42}$ For example, Zheng et al. constructed the POSSPNIPAM hydrogels via physical crosslinking using POSS-capped PNIPAM telechelics. ${ }^{41}$ Here, the PNIPAM based hydrogels were prepared via chemical photo-crosslinking with Irgacure 2959 as an initiator and OMAPOSS as a cross-linker. To increase the biocompatibility of the POSS-PNIPAM hydrogel for cell adhesion, a small amount of oligo-ethylene glycol methacrylate (OEGMA) was introduced to hybrid hydrogel. Meanwhile, the hydrophilic OEGMA was copolymerized into the hybrid hydrogel to counteract the hydrophobicity of the network resulted from hydrophobic POSS. After the polymerization, the POSSPNIPAM hydrogels were still soaked in 1,4-dioxane for 2 days to remove the unreacted components. When the solvent was replaced with phosphate buffer saline at room temperature (18 ${ }^{\circ} \mathrm{C}$ ), the POSS-PNIPAM hydrogels showed totally different swelling behavior. They slightly shrunk from the solvent of dioxane to PBS, due to the hydrophobic nature of POSS moieties. Moreover, the pure PNIPAM hydrogel (MN) is still transparent, while the transparency of POSS-PNIPAM hybrid hydrogels gradually decreases with the increase of POSS content. The swelling behavior is an important factor of hydrogels that is associated with transferring of nutrients, metabolic waste and ions under physiological conditions. ${ }^{43}$ The temperature-dependent swelling behavior of four PNIPAMbased hydrogels was evaluated. Since PNIPAM is hydrophilic under its LCST, these hydrogels showed relatively large swelling ratios at a low temperature (Fig. 1A and B). When the temperature was increased above the LCST, the intermolecular hydrogen bonds in hydrogels were broken, and the stretched PNIPAM chains were undergone the configuration reversal to entangled arrangements, leading to the shrinkage of the hydrogels. However, the PNIPAM-based hydrogels did not show the sharp conformational transition beside the P10N hydrogel, which is associated to their crosslinking structure. The chain length of PNIPAM between two gel knots in the crosslinking structure become much shorter with the increase with crosslinking density. Moreover, the swelling ratio is also influenced by the crosslinking density, and it can be seen that the lower swelling ratio for the hydrogels with a higher crosslinking density. Thus, P10N with a low crosslinking density has a highest swelling ratio, although it was prepared using hydrophobic POSS as a crosslinking agent. Fig. S2 $\uparrow$ presented the swelling kinetics of the PNIPAM hydrogels at $4{ }^{\circ} \mathrm{C}$. It could be clearly seen that the absorption swelling behavior of PNIPAM hydrogels is similar to that of their temperature-responsive
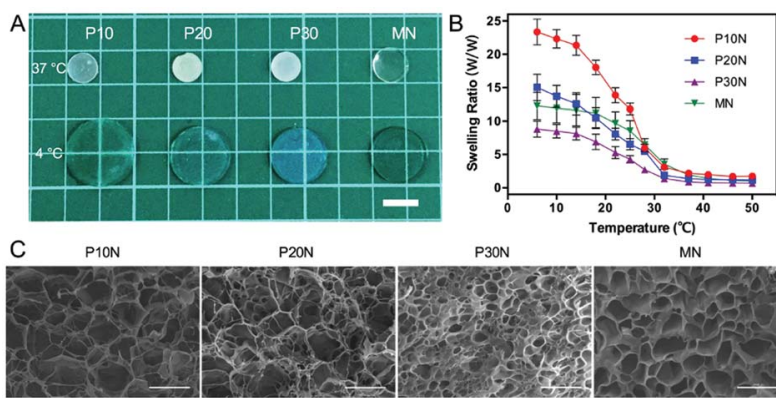

P3ON

MN

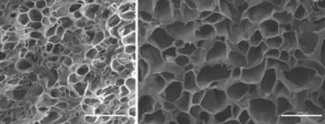

Fig. 1 (A) The bright field pictures of PNIPAM-based hydrogels soaked in PBS at $37{ }^{\circ} \mathrm{C}$ (up) and $4{ }^{\circ} \mathrm{C}$ (bottom), scale bar: $10 \mathrm{~mm}$. (B) The temperature-responsive equilibrium swelling ratios $\left(4\right.$ to $\left.50^{\circ} \mathrm{C}\right)$ and $(\mathrm{C})$ the SEM images of PNIPAM based hydrogels. $500 \times$, scale bar: $50 \mu \mathrm{m}$.

deswelling properties. The P10N showed relatively fast water absorption rate, this could be attributed to the low crosslinking density.

The microstructure of PNIPAM hydrogels was characterized by SEM. All PNIPAM-based hydrogels displayed continuous and ordered porous structures, which exhibited homogeneous architecture. For POSS-PNIPAM hybrid hydrogels, their average pore diameter decreased with the increase of POSS content, where P10N had a larger size in range of $20-40 \mu \mathrm{m}$ and P30N had a smaller size of 6-10 $\mu \mathrm{m}$ (Fig. 1C). Similarly, the thickness of network walls was also increased with the increasing POSS content. In addition, the pore size and water absorption of the hydrogel networks were positively correlated, which was corresponding to the result of temperature-dependent swelling behavior above. Comparing the microstructure of the pure PNIPAM hydrogel (MN) with P30N, the MN hydrogel had a bigger pore size and thinner network wall, although these hydrogels had a similar crosslinking density.

The thermal stability of PNIPAM based hydrogels were investigated by thermogravimetric analysis (TGA) in a nitrogen atmosphere. The TGA curves were shown in Fig. $\mathrm{S} 3, \dagger$ we could observe a subtle mass drop located at $T \sim 100{ }^{\circ} \mathrm{C}$, which was attributed to the elimination of the moisture in PNIPAM films. All the POSS-PNIPAM hydrogels showed a similar thermal stability, where the degradation occurred at $320-330{ }^{\circ} \mathrm{C}$, and the most weight loss was at about $440{ }^{\circ} \mathrm{C}$. Compared to the $\mathrm{MN}$ hydrogel, POSS-containing hydrogels possessed a higher initial thermal decomposition temperature and a higher most weight loss temperature, which indicated that the incorporation of the POSS moiety effectively improved the thermal stability of PNIPAM hydrogels. Besides, when the temperature rose to $800{ }^{\circ} \mathrm{C}$, the organic components were thermal decomposed, and the residual ash was attributed to the ceramics of POSS segments and the carbon black. The residual ash of P10N, P20N, P30N and $\mathrm{MN}$ hydrogel was $8.81 \mathrm{wt} \%, 9.62 \mathrm{wt} \%, 10.95 \mathrm{wt} \%$ and $5.25 \mathrm{wt} \%$ (at $800{ }^{\circ} \mathrm{C}$ ), respectively, which was agreeable with the amount of POSS in the pre-gel solutions.

The mechanical property was one of the key parameters of hydrogels in tissue engineering. The storage modulus $\left(\mathrm{G}^{\prime}\right)$ and loss modulus $\left(\mathrm{G}^{\prime \prime}\right)$ of PNIPAM-based hydrogels as a function of frequency range from $10^{-1} \mathrm{~Hz}$ to $10^{1} \mathrm{~Hz}$ were shown in Fig. 2A. All PNIPAM hydrogels exhibited a solid-like behavior $\left(\mathrm{G}^{\prime}>\mathrm{G}^{\prime \prime}\right)$, 

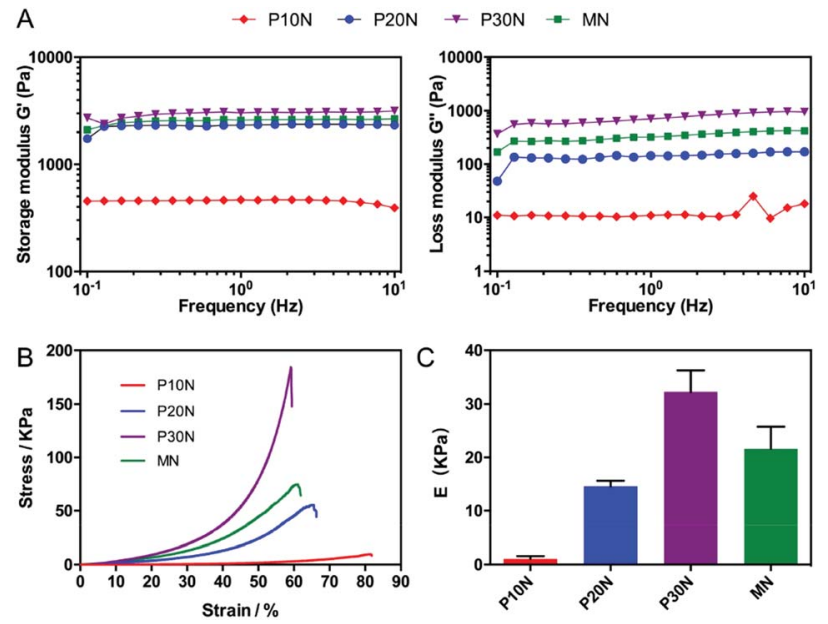

Fig. 2 (A) Storage modulus $\left(G^{\prime}\right)$ and loss modulus $\left(G^{\prime \prime}\right)$ of PNIPAM based hydrogels. (B) Stress-strain curve and (C) elastic modulus of PNIPAM based hydrogels from unconfined compressive test. Results were mean $\pm \mathrm{SD}, n=3$

and their $\mathrm{G}^{\prime}$ values were almost independent of the frequency, which proved that the hydrogels had been well crosslinked. Moreover, within the swept frequency range, the $\mathrm{G}^{\prime}$ of P10N and $\mathrm{P} 20 \mathrm{~N}$ was an order greater than their $\mathrm{G}^{\prime \prime}$, indicating these two kind of POSS-PNIPAM gels had the characteristics of an elastomer. ${ }^{4}$ In addition, as expected, P30N had both the highest $\mathrm{G}^{\prime}$ and $\mathrm{G}^{\prime \prime}$ values among these tested hydrogels, and it had the character of rigid materials. To better understand the effect of POSS on the mechanical properties, we carried out uniaxial compressive measurements on these PNIPAM-based hydrogels. The stress-strain curves and elastic modulus of different hydrogels were shown in Fig. 2B and C, respectively. With the increase of POSS content, both the critical fractured stress and the elastic modulus of POSS-PNIPAM hydrogels increased, while the ultimate strain decreased, which was consistent with the rheological results. P30N had a similar ultimate strain as the MN hydrogel $(59.2 \%$ and $60.7 \%$, respectively), but the critical fractured stress and the elastic modulus of P30N were significantly higher than that of the MN hydrogel, which embodied the advantages of POSS as a crosslinker. It was noted that the mechanical properties of hydrogels were closely associated to the content of inorganic components. ${ }^{45,46}$ The good mechanical performance of PN hydrogels could be attributed to the inorganic POSS-enhanced role and the unique cross-linked structure provided by POSS moieties. However, excessively addition of POSS would reduce the distance of adjacent crosslink points, thereby reducing the effect of chain entanglement, which caused the hydrogels to become brittle.

\subsection{In vitro cell culture of hAMSCs on POSS-PNIPAM hybrid hydrogels}

To evaluate the potential of POSS-PNIPAM hydrogels in culturing anchorage dependent cells, human amniotic mesenchymal stem cells (hAMSCs) were used as seeding cells with a cell density of $2.5 \times 10^{4}$ cells per $\mathrm{cm}^{2}$. We measured the
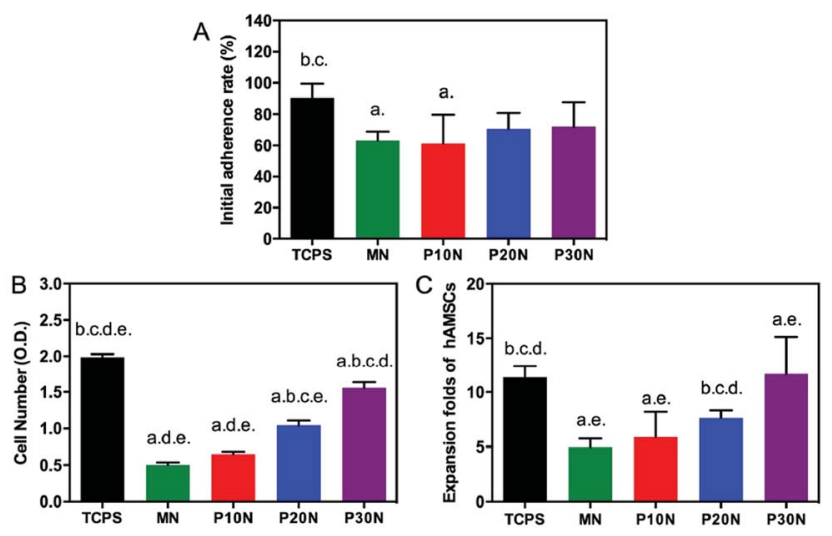

Fig. 3 (A) The initial adherent rate of hAMSCs on PNIPAM-based hydrogels and TCPS; (B) the total cell number and (C) the cell expansion folds on $4^{\text {th }}$ day; $p<0.05$, and $a, b, c, d$, e represent compare to TCPS, MN, P10N, P20N and P30N, respectively; $n=3$.

attachment ratio of hAMSCs on PNIPAM based hydrogels, which was obtained after $12 \mathrm{~h}$ of cell incubation. Clearly, the initial cell attachment ratios on MN and P10N hydrogels were all about $60 \%$, which were significantly lower than that on TCPS, while on P20N and P30N hydrogels, more than 70\% seeding cells attached after $12 \mathrm{~h}$ of cultivation (Fig. 3A). In the previous works, the cell attachment was promoted mostly by using bioactive factors like $\mathrm{Rgd},{ }^{28}$ fibronectin ${ }^{22}$ or collagen. ${ }^{47}$ However, these bioactive factors were extremely expensive, which was not conducive to the industrialization of thermosensitive materials.

The cell proliferation on PNIPAM-based hydrogels was evaluated on day 4 by cell counting kit-8 assay (Fig. 3B and C). Obviously, the cell density gradually increased with the increase of POSS content, and the P30N hydrogel had the highest cell number over P10N, P20N and MN hydrogels. Previous studies had reported that stiffness was an important factor to affect cell proliferation, that the increased modulus of substrates promoted cell proliferation. ${ }^{48}$ It is very interesting that P20N had a lower modulus than MN, but the cell number on P20N was more than that on MN. This is because the cell growth on substrates was a complicated process, which was also determined by other factors. In this work, the POSS was incorporated into hybrid hydrogels not only enhanced their mechanical property, but improved other properties such as hydrophobicity and biocompatibility, so that the P30N exhibited the best effect on supporting cell growth among these hydrogels. In addition, the cellular morphologies on different types of surfaces, including P10N, P20N, P30N, MN and TCPS, were observed by immunofluorescence staining (rhodamine-phalloidin \& DAPI) after 4 days of cultivation (Fig. 4). Notably, hAMSCs showed a good adhesion capacity, which was evidenced by that wellstretched F-action could be clearly observed on TCPS and all PNIPAM-based hydrogels except MN. Moreover, the cells exhibited adequate spreading on the P30N hydrogel and reached about $80 \%$ confluence, while there was only small amount of scattered cells attached on MN hydrogel in round shape. This result revealed that hAMSCs could well-attach on 


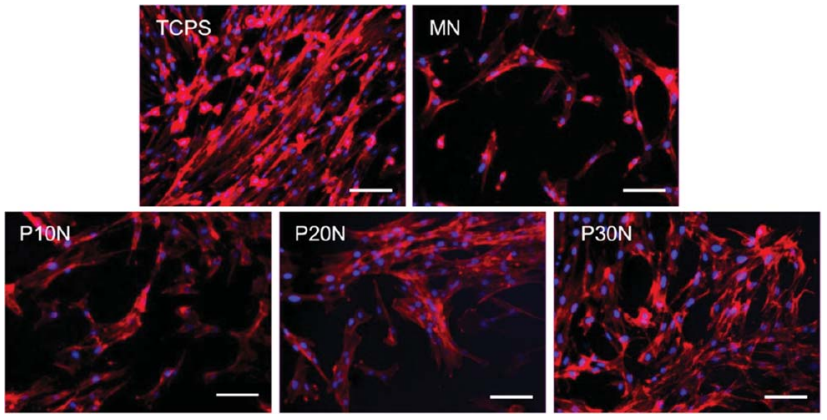

Fig. 4 Fluorescent images stained with rhodamine-phalloidin (red) and DAPI (blue) of hAMSCs on different substrates. Scale bar: $100 \mu \mathrm{m}$.

POSS-based hydrogels, which further proved that incorporation of POSS could efficiently improve the biocompatibility of hydrogels.

The cell harvest was achieved by transferring the hydrogel films to fresh $\alpha$-MEM medium at $4{ }^{\circ} \mathrm{C}$ (below the LCST) for $40 \mathrm{~min}$. Cells detached from all types of the experimental hydrogels, and the process of cell detachment was also observed by using an inverted microscope. After lowering the medium temperature, the cells cultured on P30N (in the dotted circle) gradually turned from elongated shape into round form (Fig. 5), which indicated the interaction between the cells and the hydrogel surface changed. Additionally, since P30N absorbed more water at lower temperature, the distance between the neighbouring cells in the dotted circle increased. After $40 \mathrm{~min}$, the cells completely detached from the surface of P30N hydrogels at low temperature. The detachment efficiency and the viability of harvested cells were determined by hemocytometer and trypan blue, respectively. As shown in Table 1, the detachment ratio of hAMSCs on P10N, P20N and P30N was similar (about 70\%), indicating that POSS had little impact on cell

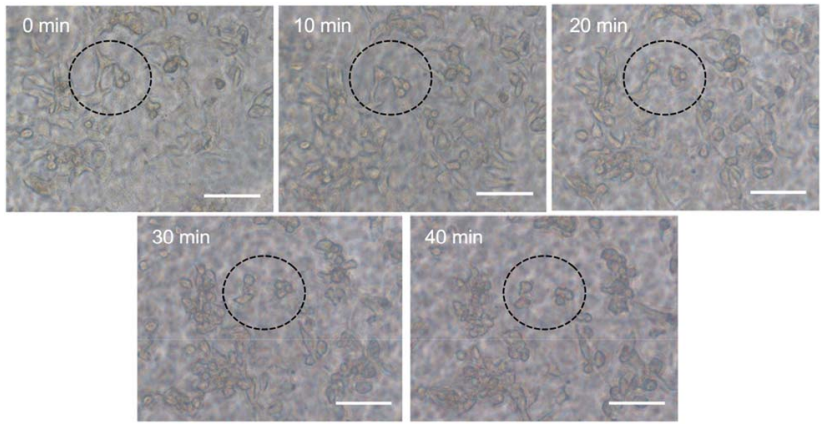

Fig. 5 Time lapse imaging tracked the morphology changing of individual cells on P30N hydrogels after cold treatment. Scale bar: $100 \mu \mathrm{m}$.

Table 1 The harvesting rate and the viability of the harvested cells on hydrogels

\begin{tabular}{llllll}
\hline Code & TCPS & MN & P10N & P20N & P30N \\
\hline Harvest ratio & $5.8 \%$ & $47.6 \%$ & $65.6 \%$ & $72.3 \%$ & $68.0 \%$ \\
Viability & N/A & $91.2 \%$ & $92.9 \%$ & $94.4 \%$ & $90.6 \%$
\end{tabular}

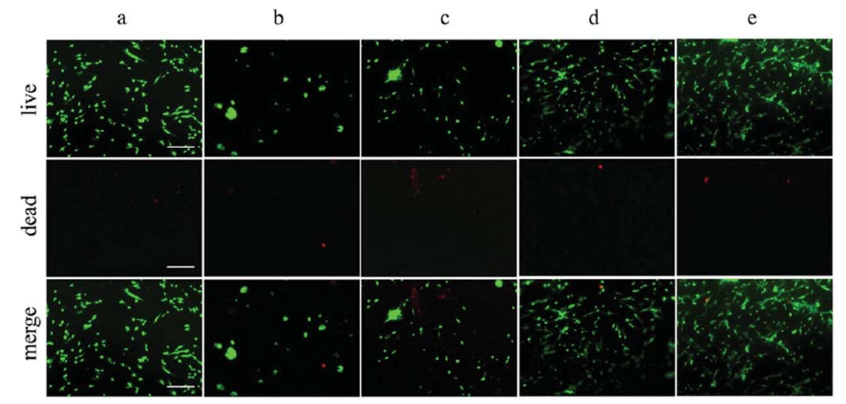

Fig. 6 Live/dead assay of human amniotic mesenchymal stromal cells on (a) TCPS, (b) MN hydrogels, (c) P10N hydrogels, (d) P20N hydrogels and (e) P30N hydrogels. (Green: live; Red: dead); scale bar $=200 \mu \mathrm{m}$.

harvest. However, only half of the cells detached on MN hydrogel and almost no cell released from TCPS after cold treatment. The living cell ratio of the cells harvested from all hydrogels was greater than $90 \%$, which indicated the harvested cells were of high activity. The harvested cells were re-seeded on TCPS and PNIPAM based hydrogels at the original seeding density $\left(2.5 \times 10^{4}\right.$ cells per $\left.\mathrm{cm}^{2}\right)$, respectively, and the cell viability was determined by live \& dead staining after cultivation for 24 h. As shown in Fig. 6, a high proportion of living cells could be clearly seen on three types of POSS-PNIPAM hydrogels and TCPS, indicating that short-term cold treatment had little effect on cell viability. However, the cells did not show obvious adhesion behavior on MN hydrogels, they only aggregated in round shape. The results above demonstrated that the POSSPNIPAM hydrogels were biocompatible platforms for cell culture and harvest. In order to study the effect of cold treatment on cellular re-proliferation ability, cells harvested from P30N hydrogels and TCPS were re-seeded on the P30N hydrogels and TCPS at the original density $\left(2.5 \times 10^{4}\right.$ cells per $\left.\mathrm{cm}^{2}\right)$, respectively. The cells were cultured on TCPS and P30N for three successive passages. For each passage, the expansion fold of cells on TCPS was slightly higher than that on P30N hydrogels (Fig. S4 $\dagger$ ), but there was no significant difference, which demonstrated that the process of harvesting cells from P30N hydrogels by cooling treatment almost did not affect their reproliferation ability.

\section{Conclusions}

In summary, we have successfully constructed thermosensitive POSS-PNIPAM hydrogels via one-pot crosslinking reaction by using POSS as a crosslinker. POSS-PNIPAM hybrid hydrogels demonstrated the enhanced thermal stability and the mechanical properties by introduction of POSS, which also increased with the content of POSS increasing. The results of the in vitro experiments showed that hAMSCs could well attach and proliferate on the POSS-PNIPAM hybrid hydrogels. More importantly, the proliferated hAMSCs cells were easily detached from the surface of POSS-PNIPAM hybrid hydrogels under low temperature without damage. Hence, POSS-PNIPAM hydrogel system provides a bright prospect for cell sheet engineering in regenerative medicine. 


\section{Conflicts of interest}

There are no conflicts to declare.

\section{Acknowledgements}

This work was financially supported by the National Natural Science Foundation of China (grant number 21574039), the Science and Technology Commission of Shanghai Municipality (grant number 15JC1401402), and the Open Funding Project of the State Key Laboratory of Bioreactor Engineering.

\section{Notes and references}

1 J. Shi, Y. Jiang, X. Wang, H. Wu, D. Yang, F. Pan, Y. Su and Z. Jiang, Chem. Soc. Rev., 2014, 43, 5192-5210.

2 K. Shah, Adv. Drug Delivery Rev., 2012, 64, 739-748.

3 M. C. Serrano, M. C. Gutiérrez and F. del Monte, Prog. Polym. Sci., 2014, 39, 1448-1471.

4 D. Seliktar, Science, 2012, 336, 1124-1128.

5 H. E. Canavan, X. Cheng, D. J. Graham, B. D. Ratner and

D. G. Castner, J. Biomed. Mater. Res., Part A, 2005, 75, 1-13.

6 H. E. Canavan, X. Cheng, D. J. Graham, B. D. Ratner and D. G. Castner, Plasma Processes Polym., 2010, 3, 516-523.

7 D. G. Phinney and M. F. Pittenger, Stem Cells, 2017, 35, 851858.

8 D. C. Ding, Y. H. Chang, W. C. Shyu and S. Z. Lin, Cell Transplant., 2015, 24, 339-347.

9 M. Krogsgaard, M. A. Behrens, J. S. Pedersen and H. Birkedal, Biomacromolecules, 2013, 14, 297-301.

10 M. Chen, X. He, K. Wang, D. He, S. Yang, P. Qiu and S. Chen, J. Mater. Chem. B, 2013, 2, 428-436.

11 X. Lin, D. Tang, Z. Yu and Q. Feng, J. Mater. Chem. B, 2014, 2, 651-658.

12 G. Pan, Q. Guo, Y. Ma, H. Yang and B. Li, Angew. Chem., Int. Ed., 2013, 52, 6907-6911.

13 Q. Zhang, P. Dong, L. Chen, X. Wang and S. Lu, J. Biomed. Mater. Res., Part A, 2014, 102, 76-83.

14 L. S. Cheung, X. Zheng, A. Stopa, J. C. Baygents, R. Guzman, J. A. Schroeder, R. L. Heimark and Y. Zohar, Lab Chip, 2009, 9, 1721-1731.

15 N. Vanparijs, L. Nuhn and B. G. De Geest, Chem. Soc. Rev., 2017, 46, 1193-1239.

16 I. C. Peng, C.-C. Yeh, Y.-T. Lu, S. Muduli, Q.-D. Ling, A. A. Alarfaj, M. A. Munusamy, S. S. Kumar, K. Murugan, H.-c. Lee, Y. Chang and A. Higuchi, Biomaterials, 2016, 76, 76-86.

17 J. Gan, X. X. Guan, J. Zheng, H. Guo, K. Wu, L. Liang and M. Lu, RSC Adv., 2016, 6, 32967-32978.

18 L. Hou and P. Wu, Soft Matter, 2015, 11, 2771-2781.

19 J. Heyda, S. Soll, J. Yuan and J. Dzubiella, Macromolecules, 2013, 47, 2096-2102.

20 T. Okano, N. Yamada, M. Okuhara, H. Sakai and Y. Sakurai, Biomaterials, 1995, 16, 297-303.

21 B. Guo, G. Pan, Q. Guo, C. Zhu, W. Cui, B. Li and H. Yang, Chem. Commun., 2015, 51, 644-647.
22 Q. Yu, L. M. Johnson and G. P. López, Adv. Funct. Mater., 2014, 24, 3751-3759.

23 M. J. Perezroldan, D. Debarnot and F. Poncinepaillard, RSC Adv., 2014, 4, 31409-31415.

24 P. Viswanathan, M. G. Ondeck, S. Chirasatitsin, K. Ngamkham, G. C. Reilly, A. J. Engler and G. Battaglia, Biomaterials, 2015, 52, 140-147.

25 J. Mitra, G. Tripathi, A. Sharma and B. Basu, RSC Adv., 2013, 3, 11073-11094.

26 Y. Qiu, A. C. Brown, D. R. Myers, Y. Sakurai, R. G. Mannino, R. Tran, B. Ahn, E. T. Hardy, M. F. Kee and S. Kumar, Proc. Natl. Acad. Sci. U. S. A., 2014, 111, 14430-14435.

27 S. Han and C. C. Lin, J. Mater. Chem. B, 2016, 4, 4969-4974. 28 G. Pan, B. Guo, Y. Ma, W. Cui, F. He, B. Li, H. Yang and K. J. Shea, J. Am. Chem. Soc., 2014, 136, 6203-6206.

29 Y.-S. Chen, P.-C. Tsou, J.-M. Lo, H.-C. Tsai, Y.-Z. Wang and G.-H. Hsiue, Biomaterials, 2013, 34, 7328-7334.

30 D. N. Heo, N. J. Castro, S. J. Lee, H. Noh, W. Zhu and L. G. Zhang, Nanoscale, 2017, 9, 5055-5062.

31 C. Lo, D. Zhu and H. Jiang, Soft Matter, 2011, 7, 5604-5609. 32 W. Zhang and A. H. E. Müller, Prog. Polym. Sci., 2013, 38, 1121-1162.

33 X. Pan, H. Gao, G. Fu, Y. Gao and W. Zhang, $R S C A d v .$, 2016, 6, 23471-23478.

34 W. Jian, Q. Ge and P. T. Mather, Macromolecules, 2010, 43, 7637-7649.

35 B. Yu, X. Jiang, N. Qin and J. Yin, Chem. Commun., 2011, 47, 12110-12112.

36 C. Shen, Y. Han, B. Wang, J. Tang, H. Chen and Q. Lin, RSC Adv., 2015, 5, 53782-53788.

37 D. K. Wang, S. Varanasi, E. Strounina, D. J. T. Hill, A. L. Symons, A. K. Whittaker and F. Rasoul, Biomacromolecules, 2014, 15, 666-679.

38 M. F. Griffin, R. G. Palgrave, A. M. Seifalian, P. E. Butler and D. M. Kalaskar, Biomater. Sci., 2015, 4, 145-158.

39 M. Wang, Y. Zhou and W. S. Tan, Biotechnol. Bioprocess., 2010, 15, 1047-1058.

40 X. Hou, W. Yang, A. Li, J. Hou and C. Zhang, Polym. Bull., 2017, 74, 1831-1847.

41 L. Wang, K. Zeng and S. Zheng, ACS Appl. Mater. Interfaces, 2011, 3, 898-909.

42 K. Zeng, L. Wang and S. Zheng, J. Phys. Chem. B, 2009, 113, 11831-11840.

43 Y. N. Zhang, R. K. Avery, Q. Vallmajo-Martin, A. Assmann, A. Vegh, A. Memic, B. D. Olsen, N. Annabi and A. Khademhosseini, Adv. Funct. Mater., 2015, 25, 4814-4826. 44 X. Hu, D. Li, F. Zhou and C. Gao, Acta Biomater., 2011, 7, 1618-1626.

45 C. Chung, B. L. Pruitt and S. C. Heilshorn, Biomater. Sci., 2013, 1, 1082-1090.

46 Z. Ren, Y. Zhang, Y. Li, B. Xu and W. Liu, J. Mater. Chem. B, 2015, 3, 6347-6354.

47 J. D. Kim, J. S. Heo, T. Park, C. Park, H. O. Kim and E. Kim, Angew. Chem., 2015, 127, 5967-5971.

48 Y. Li, Y. Zhang, S. Feng, T. Lei, Y. Wei and W. Xing, Colloids Surf., B, 2016, 149, 168-173. 